\title{
El dilema de lo orgánico y lo social en las emociones deportivas
}

\section{The dilemma of physiological and social emotions in sports}

\author{
JuAn Luis Yuste Lucas* \\ jlyuste@um.es \\ José Ignacio Alonso Roque* \\ jialonso@um.es \\ Gemma María Gea García** \\ gmgea@ucam.edu \\ Nuria Ureña Ortín* \\ nuriaur@um.es \\ * Universidad de Murcia, España \\ ** Universidad Católica San Antonio de Murcia, España
}

\section{Resumen:}

La relación entre las emociones y variables fisiológicas es un hecho cada vez más investigado en el ámbito físico-deportivo. Así, el presente trabajo, fruto de una revisión bibliográfica, tiene como objetivo analizar aquellos estudios que han acometido investigaciones sobre posibles correlaciones, positivas o negativas, habidas entre las emociones y parámetros biológicos. En este sentido, y a nivel hormonal, se analiza la relación existente entre el cortisol y el estrés psicofisiológico, donde dicha hormona es un factor que puede ser utilizado como un indicador del estado emocional, y en el que una prolongada situación de emocional negativa puede conllevar a un estado de enfermedad pisco-física. Del análisis sobre la actividad electroencefalográfica y las emociones, apuntar una estrecha relación entre ambas, llegándose a construir verdaderas bases de datos de emociones como consecuencia de señales fisiológicas. Por otra parte, también encontramos estrechos la-

\begin{abstract}
:
The link between the emotions and the physiological variables is increasingly researched by the field of study of Physical Education and Sport. The aim of this work, which is the result of a bibliographic review, is to analyze those studies researching the possible correlations, both positive and negative, between the emotions and the biological parameters. In this sense, at a hormonal level, this work will analyze the relationship between cortisol and the psychophysiological stress, where this hormone is a factor that can be used as an indicator of the emotional state, and where a prolonged negative emotional state can entail psychophysical disorders. Regarding the analysis on the electroencephalographic activity and the emotions, it must be noticed the close relationship between them - we can create reliable databases of emotions resulting from physiological signs. We also find close links between the heart rate variability and the mood. Finally, we analyze works that
\end{abstract}


El dilema de lo orgánico y lo social en las emociones deportivas

José Ingnacio Alonso Roque, Juan luis Yuste lucas, Gemma María Gea García y Nuria

UREÑA OrTín

zos entre la variabilidad de la frecuencia cardíaca y el estado de ánimo. Por último, también se analizan trabajos que han investigado sobre la relación entre la composición corporal y el psicotipo, hallando una correlación entre emoción positiva y tipo de somatotipo.

Palabras clave:

Emociones, variabilidad de la frecuencia cardíaca, cortisol, somatotipo. study the relationship between the body composition and the mental identity, and we find a correlation between the positive emotion and the type of somatotype.

\section{Key words:}

Emotions, heart rate variability, cortisol, somatotype.

\section{Résumé:}

La relation entre les émotions et les variables physiologiques est un fait de plus en plus étudié dans le domaine physico-sportif. ainsi, le présent travail, le résultat d'une analyse documentaire, vise à analyser les études qui ont entrepris des enquêtes sur les corrélations possibles, positives ou négatives, eu entre les émotions et les paramètres biologiques. dans ce sens, et a niveau hormonal, on analyse la relation existante entre le cortisol et le stress psychophysiologique, dans lequel ladite hormone est un facteur qui peut être utilisé comme indicateur de l'état émotionnel, et dans laquelle une situation prolongé d'émotion négative peut conduire à un état de maladies psycho-physiques. de l'analyse sur l'activité électroencéphalographique et les émotions, noter une étroite relation entre elles, arrivant à construire une véritable base de donnée d'émotions par conséquence de signaux physiologiques. d'un autre coté, on trouve aussi des liens étroits entre la variabilité de la fréquence cardiaque et l'état de l'humeur. enfin, on analyse aussi des travaux de recherche sur la relation entre la composition corporelle et psychotype, en trouvant une corrélation entre émotion positive et type de somatotype.

\section{Mots clés :}

Émotions, Variabilité de la fréquence cardiaque, cortisol, somatotype

Fecha de recepción: 16-1-2014

Fecha de aceptación: 30-1-2014

\section{El dilema de lo orgánico y lo social en las emociones deportivas}

Las investigaciones que abordan trabajos sobre emociones y variables fisiológicas están ocupando un gran protagonismo en el ámbito de la actividad física, en el que cada vez se hace más imprescindible establecer relaciones entre el estado emocional y determinadas reacciones fisiológicas. A continuación, presentamos evidencias científicas que acometen trabajos para hallar dichas relaciones. 


\section{Estado emocional y funcionamiento orgánico}

Actualmente, las investigaciones relacionadas con las emociones están centradas en variables biológicas, conductuales y cognitivas (Palmero, 1996 y Barra, 2003). Así, y como indica Ramos y cols. (2009), una acertada definición de emoción debería indicar las más importantes funciones de esta (adaptación orgánica, comunicación social y experiencia subjetiva de la persona implicada). En esta línea, en 1992, Oatley señala que una emoción es una experiencia que conlleva implícita tres sistemas de respuesta (cognitivo-subjetivo; conductual-expresivo y fisiológico-adaptativo). Por otra parte, Vidales (1978) señala que son varios los parámetros orgánicos que pueden hacer notar la presencia de una emoción (la piel, distribución de la sangre, ritmo cardíaco, respiración, respuesta pupilar, secreción salival, movilidad gastrointestinal, etc.).

Tal y como indican Ramos, Piqueras, Martínez y Oblitas (2009), la teoría más antigua sobre las emociones corresponde a James-Lange a finales del siglo XIX, indicando que estas se fundamentaban en la percepción de un estímulo por parte del sujeto, que seguía con la experiencia de la emoción (la experiencia de una emoción lleva consigo una serie de variables que influye para que una persona experimente una emoción de forma particular en base de sus experiencias, aprendizajes, carácter, etc.) y, por último, terminaba con una respuesta (modificaciones fisiológicas en nuestro organismo) al estímulo recibido, siendo las emociones el resultado de estos. Al respecto, en 1934, Duffy indica que toda emoción consiste en la movilización de energía del organismo.

Papez (1937) señala que ciertas partes del sistema nervioso (hipotálamo y sistema límbico) son las responsables bioquímicas de todo tipo de experiencia emocional. Por otra parte, MacLean (1975) apunta al sistema límbico como principal responsable de dichas experiencias emocionales. En esta línea, Olds y Milher (1954) señalan que el hipotálamo y el tronco del encéfalo son las zonas del sistema nervioso generadoras de placer cuando estás son estimuladas, o activadas, siendo la dopamina el neurotransmisor encargado de suministrar los sentimientos de placer y refuerzo que motivarán a una persona a realizar ciertas actividades (Wise, 1982), y donde Lyndsey (1951) y Duffy (1972) señalan la transcendencia de dicha activación para que se den las emociones. Por otra parte, cuando el hipotálamo (parte del cerebro encargada de regular las 
emociones) es extirpado desaparece totalmente la conducta emocional (Whittaker, 1987).

Sobre la etiología de la depresión y sus síntomas, inicialmente se prestó más atención en el papel realizado por la serotonina y norepinefrina para la comprensión de la misma; sin embargo, en la actualidad se inclinan más al papel de la dopamina para dicha comprensión, donde el sistema dopaminérgico lleva a cabo una importante función en la pérdida de motivación, a la vez que los circuitos neuronales relacionados con la depresión también regulan las emociones (Márquez y Garatachea, 2013). Por otra parte, no debemos olvidar que la secreción de la hormona cortisol, la cual es generada por la activación del eje hipotalámico-pituitaria-adrenal (HPA), es consecuencia de la aparición de amenaza real o autopercibida (Kalat, 2004); siendo por tanto una respuesta adaptativa frente a una situación de estrés; sin embargo, si la activación del eje HPA es prolongada en el tiempo, esta puede conllevar serios problemas para la salud (depresión, ansiedad, etc.) (Pinna, Van Den Bergh, Van Calster y Van Huffel, 2008).

Tal y como indica Silverthorn (2009), es difícil definir los estados de ánimo a nivel neurológico, pero la evidencia obtenida en el estudio y el tratamiento de los trastornos del estado de ánimo indica que lo que en otra época se atribuía a un origen puramente psicológico es en realidad un trastorno funcional del SNC, donde la depresión y otros trastornos del estado de ánimo son el resultado de la liberación anormal de neurotransmisores o su recepción alterada en diferentes regiones encefálicas

Por otra parte, el cortisol es utilizado para el seguimiento de la respuesta al estrés durante la práctica deportiva (Michaux y Robert, 2004). Sin embargo, la respuesta de esta hormona puede verse alterada en función del tipo de ejercicio (Passelergue, Robert y Lac, 1995), nivel y tipo de entrenamiento (Tremblay, Copeland, y Van Helder, 2004), condiciones climatológicas (Starkie, Hargreaves, Roland y Febbraio, 2005), entre otros. Es evidente que, aun hallando disparidad en los resultados sobre niveles de cortisol en la práctica físico-deportiva, el estudio de esta hormona sigue siendo interesante porque refleja la influencia física y psicológica, dualidad que es necesaria analizar. En esta línea, diferentes investigaciones (Bouget, Rouveix, Michaux, Pequignot y Filaire, 2006; Doan, Newton, Kraemer, Kwon y Scheet, 2007; Minetto et al., 2007; Crewther, Cronin, Keogh y Cook, 2008; Beaven, Gill y Cook, 2008; Moreira, Arsati, de Oliveira, da Silva y de Arau' jo, 2009, Lusa Cadore et 
al., 2009; Heijsman et al., 2012) llevadas a cabo en diferentes edades y prácticas físico-deportivas, señalan que la relación entre los niveles de cortisol salival y el estrés psicofisiológico, aportan una herramienta indicadora de auto-reporte psicológico y cambios de los niveles de ansiedad relacionados con los éxitos en la práctica del ejercicio físico. Al respecto, Hanton, Thomas y Maynard (2004) indican que el mismo nivel de ansiedad puede ser autopercibido por deportistas bien como facilitadores o como debilitadores respecto a su rendimiento deportivo. En esta línea, el trabajo realizado por Filaire, Alix, Ferrand y Verger (2009) en tenistas durante competición, señala que el día de la competición las mujeres desprendieron valores de cortisol significativamente superiores a los hombres, a la vez que estas presentaron niveles superiores de ansiedad somática respecto a los hombres, así como también se hallaron valores de cortisol significativamente superior en los perdedores respecto a los ganadores, y donde los ganadores mostraron menor ansiedad cognitiva y mayor registro de auto-confianza respecto a los perdedores.

El estudio realizado por Schellign, Calleja y Terrado (2013) en jugadores de baloncesto de élite, analizó la respuesta de la testosterona total, el cortisol y la ratio testosterona total-cortisol y su relación con el estado emocional, analizado este mediante la puntuación directa de cada sub-escala (vigor, fatiga, agresividad, tensión, depresión y confusión) del cuestionario POMS (Profiles of mood states), y donde los resultados señalan una correlación positiva con de los niveles del cortisol en sangre con la puntuación total y con tres de las sub-escalas del POMS (la tensión, la confusión, la depresión y la agresividad), por lo que el cortisol indica una correlación positiva con el estrés psicológico (Fukuda y Morimoto, 2001), por tanto, el cortisol está correlacionado positivamente con los estados de ánimo negativos.

Como señala Barra (2003), para evaluar el efecto que tiene el estado emocional en la salud física, es necesario tener presente una serie factores (fisiológico, cognitivo, social y conductual) indicados anteriormente. Al respecto, las experiencias negativas pueden llegar a ser una variable que alargue las infecciones adquiridas y retrasar la cicatrización de heridas (Kiecolt-Glaser, McGuire, Robles y Glaser, 2002), así como hacer al individuo más vulnerable a diversas enfermedades (Salovey, Rothman, Detweiler y Steward, 2000). En esta línea, Cohen y Pressman (2006) señalan que tener vivencias afectivas negativas de manera continuada como la ansiedad, depresión y hostilidad se correlacionan de manera 
positiva con la posibilidad de padecer enfermedades y con el riesgo de mortalidad; sin embargo, las vivencias positivas conllevan un menor riesgo de mortalidad, disminución de síntomas de enfermedad y diminución del dolor. Además, cuando los individuos son expuestos a un virus respiratorio en el laboratorio, se observa que aquellos que experimentan mayor ánimo negativo desarrollan síntomas más severos que los individuos cuyo estado de ánimo es más positivo (Cohen et al., 1995), y las personas que se enfrentan a estresores severos por un tiempo prolongado son significativamente más susceptibles al resfriado común inducido experimentalmente (Cohen et al., 1998).

Aun sabiendo que la activación emocional tiene efectos inmediatos sobre el sistema inmunológico, todavía no está claro la duración de estos efectos ni tampoco si la persistencia en el estado anímico conlleva incremento significativo a la resistencia de las personas a ciertas patologías (Barra, 1993).

Debemos tener en cuenta que la práctica de actividad física se correlaciona de manera positiva con una buena percepción de estado de forma física, conlleva un positivo estado de ánimo y provoca la liberación de ciertos neurotransmisores cerebrales (serotonina, dopamina y noradrenalina) implicados en las emociones (Arruza et al., 2008). Al respecto, numerosas investigaciones señalan que llevar a cabo una práctica de actividad física bajo unos determinados parámetros de intensidad, duración y frecuencia, conllevan mejora de la salud subjetiva, estado de ánimo y la emotividad (Biddle, Fox y Boutcher, 20006), disminución de la depresión (Lawlor y Hopker, 2001), reducción de los niveles de ansiedad (Akandere y Tekin, 2005), mejora de la capacidad para afrontar situaciones estresantes (Holmes, 1993), mejora de la salud mental por la liberación de endorfinas causada por dicha práctica física, lo que a su vez conlleva la reducción de ansiedad, depresión y estrés, ya que las endorfinas actúan directamente en el cerebro provocando sensación de bienestar e inmediata relajación, inhibiendo por otra parte aquellas fibras nerviosas transmisores del dolor, lo que genera analgesia y sedación, provocando una situación de euforia a causa de la endorfina, denominándola también como la hormona de la felicidad (Martinsen, 2004; Paffenbarger, Lee y Leung, 2004), entre otros beneficios.

Tal y como señala Lyndsey (1951), existe una relación entre variables psicológicas y actividad electroencefalográfica, donde una baja activación electroencefalográfica conllevaría una baja ausencia emocional y, 
una elevada actividad electroencefalográfica implicaría una alta presencia emocional. En esta línea, indicar que las emociones han sido analizadas mediante el electroencefalograma hace más de veinte años, proceso que recoge la actividad de las neuronal de toda la zona cortical para su posterior identificación mediante modificaciones fisiológicas. Por otra parte, antes del proceso dicha identificación, se establecieron distintas características fisiológicas para cada una de las emociones que el ser humano puede manifestar (Cacioppo y Tassinary, 1990), permitiendo configurar verdaderas bases de datos de análisis de emociones haciendo uso de estas señales fisiológicas (Koelstra et al., 2011). Por otra parte, investigaciones llevadas a cabo con anterioridad, clasificaron las distintas emociones en base a la conductancia cutánea, la temperatura corporal y el ritmo cardíaco (Picard, 2000).

Wittaker (1987), indica que el electroencefalógrafo ha permitido percibir la variación de ciertos impulsos eléctricos, consiguiendo medir las descargas provenientes de las neuronas del cerebro que, en base a la emoción experimentada por la persona, la intensidad es diferente; a la vez que los factores cognoscitivos intervienen en las emociones (un individuo que se halle en una situación de la que espera experimentar una determinada emoción, manifiesta síntomas fisiológicos acordes a dicha emoción específica).

En cuanto a la variabilidad de la frecuencia cardíaca (VFC), la Medicina del Deporte propone el análisis de esta como un método válido, rápido y no invasivo para llevar a cabo la evaluación del efecto que tiene la carga de la práctica físico-deportiva sobre el organismo del deportista (Rodas, Pedret, Ramos y Capdevila, 2008); al respecto, los índices de las bandas de altas (HF) y bajas frecuencias (LF), están relacionadas con la actividad del sistema nervioso parasimpático y simpático respectivamente, donde la proporción LF/HF es utilizada como medida de equilibrio simpático-parasimpático (Task Force of the European Society of Cardiology and The North American Society of Pacing and Electrophysiology, 1996; Acharya, Joseph, Kannathal, Lim y Suri, 2006).

La información proporcionada tras el análisis de la VFC ha permitido llevar estudios de la respuesta autonómica (sistema nervioso autónomo) en situaciones de entrenamiento intenso (Leicht, Allen y Hoey, 2003; Hautala et al., 2001), de entrenamiento aeróbico (Jammes et al., 2001; Aubert, Beckers y Ramaekers, 2001; Pichot et al., 2000; Pigozzi et al., 2001), durante competiciones deportivas (Iellamo, Pigozzi, Spataro, Lu- 
cini y Pagani, 2004), durante situaciones de sobreentrenamiento deportivo (Mourot, Bouchaddi, Perrey, Rouillon y Regnard, 2004), como herramienta de seguimiento y control de una temporada de entrenamiento (Hedelin, Wiklund, Bjerle y Henriksson-Larsen, 2000), como evaluación fisiológica y cognitiva del proceso de estrés-recuperación en la preparación pre-olímpica de deportistas de elite (Cervantes, Florit, Parrado, Rodas y Capdevila, 2009), entre otros.

El cuestionario empleado para determinar el Estado de Ánimo (POMS) de Mcnair, Lorr y Droppelman (1971), ha sido utilizado para valorar el efecto del ejercicio aeróbico sobre el estado emocional (Head, Kendall, Ferner y Eagles, 1996); como variable psicológica relacionada con el sobreentrenamiento (Buchheit, Simon, Piquard, Ehrhart, y Brandenberger, 2004); como variable control de la capacidad de esfuerzo en snowboarders (Arruza, Tellechea, Arribas, Balagué y Brustad, 2005) o para investigar la relación entre el estado de ánimo medido con el POMS y la VFC en judokas (Blasco, Martínez, Baydal, Mateo y Pablos, 2010).

Otra variable fisiológica correlacionada con parámetros psicológicos, es la composición corporal. Al respecto, Sheldon (1960) relaciona el tipo de somatotipo con el psicotipo o tipo temperamental, indicando la existencia de tres tipos temperamentales: la viscerotonía (persona con predominio de la endomórfia y que se caracterizan por el gusto a una vida cómoda y fácil y en todo momento tiene una emoción positiva y sintonía con los demás); la somatotonía (persona con predominio de la mosomorfía, siempre dispuesta a tomar decisiones y enérgica, con gusto al deporte y a la competición) y la cerebrotonía (persona con predominio de la ectomorfia, dubitativas e hipersensible con una elevada represión emocional y que de forma natural tiende a ocultar sus sentimientos por miedo, dando apariencia de insensibles hasta incluso con la alegría). Al respecto, el trabajo realizado por Pailhez, Rodríguez, Ariza, Palomo y Bulbena (2009) tuvo como objetivos comparar los somatotipos de pacientes esquizofrénicos con el de un grupo control y establecer correlaciones entre el somatotipo y psicopatologías (ansiedad, tipo de esquizofrenia y gravedad clínica), los resultados apuntaron hacia una tendencia a favor de la asociación entre laxitud articular y ansiedad y entre ectomorfismo y ansiedad.

En cuanto a la relación entre rendimiento deportivo y emociones, el estudio realizado por López-Torres, Torregrosa y Roca (2007) en diez nadadoras de élite, señalan que en las mejores experiencias de rendi- 
miento se presentaron mayores niveles de flow y de emociones positivas, a la vez que se desprendían menores niveles de ansiedad. Al respecto, la composición corporal de los deportistas es una variable que está relacionada con los logros, o éxitos, deportivos (Drinkwater, Pyne y Mckenn, 2008; Fonseca-Toledo, Roquetti y Fernandes-Filho, 2010; Lago-Peña, Casais, Dellal, Rey y Domínguez, 2011; Wilber y Pitsiladis, 2012), y donde diversos estudios (Raschka y Zanellato, 2003; Bavios, Bergeles, Apostolidis, Noutsos y Koskolou, 2006; Raschka y Wolthausen, 2007; Chaabène, Hachana, Franchini, Mkaouer y Chamari, 2012) sobre la composición corporal de diferentes deportes (baloncesto, voleibol, balonmano, fútbol, kárate, máster de aeróbics de Alemania, etc.), indican que estos deportistas presentan somatotipos atléticos, asentándose en zonas mesomorfoectomorfo y ecto-mesomorfo.

\section{Referencias bibliográficas}

Acharya, U. R., Joseph, K. P., Kannathal, N., Lim, C. M., \& Suri, J. S. (2006). Heart rate variability: a review. Medical and Biological Engineering and Computing, 44, 1031-51.

Arruza, J. A., Arribas, S., Gil De Montes, L., Irazusta, S., Romero, S., \& Cecchini, J. A. (2008). Repercusiones de la duración de la Actividad Físico-deportiva sobre el bienestar psicológico. Revista Internacional de Medicina y Ciencias de la Actividad Física y el Deporte, 8(30), 171-183.

Arruza, J., Tellechea, S., Arribas, S., Balagué, G., \& Brustad, R. (2005). Capacidad de esfuerzo en snowboarders: diferencias individuales en una prueba de máximo esfuerzo en half-pipe. Revista de Psicología del Deporte, 14, 283-300.

Aubert, A.E., Beckers F., \& Ramaekers D. (2001). Short-term heart rate variability in young athletes. Journal of Cardiology, 37, 85-88.

Barra, E. (2003). Influencia del estado emocional en la salud física. Terapia psicológica, 21(1), 55-60.

Bayios, I. A., Bergeles, N. K., Apostolidis, N. G., Noutsos, K. S., \& Koskolou, M. D. (2006). Anthropometric, body composition and somatotype differences of Greek elite female basketball, volleyball and handball players. J Sports Med Phys Fitness, 46(2), 271-80.

Beaven, C. M., Gill, N. D., \& Cook, C. J. (2008). Salivary testosterone and cortisol responses in professional rugby players after four resistance exercise protocols. J Strength Cond Res, 22, 426-432.

Blasco, C., Martínez, I., Baydal, E., Mateo, M., \& Pablos, C. (2010). Relación entre el perfil de estado de ánimo (POMS) y la VFC en judokas. Cult. Cienc. Deporte, 5(13), 137.

Bouget, M., Rouveix, M., Michaux, O., Pequignot, J. M., \& Filaire, E. (2006). Relationships among training stress, mood and dehydroepiandrosterone sulphate/cortisol ratio in female cyclists. J Sports Sci, 24, 1297-1302. 
El dilema de lo orgánico y lo social en las emociones deportivas

José Ingnacio Alonso Roque, Juan luis Yuste lucas, Gemma María Gea García y Nuria

UReña Ortín

Buchheit, M., Simon, C., Piquard, F., Ehrhart, J., \& Brandenberger, G. (2004). Effect of increased training load on vagal-related indexes of heart rate variability: a novel sleep approach. American Journal of Physiology-Heart and Circulatory Physiolology, 287, 2813-2818.

Cacioppo, C. J., \& Tassinary, L. G. (1990). Inferring physiological significance from physiological signals. American Psychologist, 45(1), 16-28.

Cervantes, J. C., Florit, D., Parrado, E., Rodas, G., \& Capdevila; LI. (2009). Evaluación fisiológica y cognitiva del proceso de estrés-recuperación en la preparación pre-olímpica de deportistas de elite. Cult. Cienc. Deporte, 4(11), 111-117.

Chaabène, H., Hachana, Y., Franchini, E., Mkaouer, B., \& Chamari, K. (2012). Physical and physiological profile of elite karate athletes. Sports Med., 42(10), 829-43. doi: 10.2165/11633050-000000000-00000.

Cohen, S., \& Pressman, S. D. (2006). Positive Affect and Health. Current Direction in Psychological Science, 5(3), 122-125.

Cohen, S., Doyle, W., Skoner, D., Fireman, P., Gwaltney, J., \& Newsom, J. (1995). State and trait negative affect as predictors of objective and subjective symptoms of respiratory viral infections. Journal of Personality and Social Psychology, 68, 159-169.

Cohen, S., Frank, E., Doyle, W., Skoner, D., Rabin, B., \& Gwaltney, J. (1998). Types of stressors that increase susceptibility to the common cold in healthy adults. Health Psychology, 17, 214-223.

Crewther, B., Cronin, J., Keogh, J., \& Cook, C. (2008). The salivary testosterone and cortisol response to three loading schemes. J Strength Cond Res, 22, 250-255.

Doan, B. K., Newton R. U., Kraemer, W, J., Kwon, Y. H., \& Scheet, T. P. (2007). Salivary cortisol, testosterone, and $\mathrm{T} / \mathrm{C}$ ratio responses during a 36-hole golf competition. Int J Sports Med, 28, 470-479.

Drinkwater, E. J., Pyne, D. B., \& McKenna, M. J. (2008). Design and interpretation of anthropometric and fitness testing of basketball players. Sports Med, 38(7), 565-78.

Duffy, E. (1934). Emotion: An example of the need for reorientation in psychology. Psychological Review, 41, 184-198.

Duffy, E. (1972). Activation. En: N. S. Greenfield y R. A. Sternach (Eds.). Handbook of Psychophisiology (pp. 572-622). New York: Holt, Rinehart and Winston.

Filaire, E., Alix, D., Ferrand, C., \& Verger, M. (2009). Psychophysiological stress in tennis players during the first single match of a tournament. Psychoneuroendocrinology, 34, 150-157.

Fonseca-Toledo, C., Roquetti, P., \& Fernandes-Filho, J. (2010). Anthropometrical profile of Brazilian junior volleyball players for different sports requirement levels. Rev Salud Pública (Bogota), 12(6), 915-28.

Fukuda, S., \& Morimoto, K. (2001). Lifestyle, Stress and Cortisol Response: Review II. Environmental Health and Preventive Medicine, 6, 15-21.

Hanton, S., Thomas, O., \& Maynard, I. (2004). Competitive anxiety responses in the week leading up to competition: the role of intensity, direction and frequency dimensions. Psychol Sport Exerc, 5, 169-181.

Hautala, A., Tulppo, M.P., Makikallio, T.H., Laukkanen, R., Nissila, S., \& Huikuri, H.V. (2001). Changes in cardiac autonomic regulation after prolonged maximal exercise. Clinical Physiology, 21, 238-245. 
Head, A., Kendall, M.J., Ferner, R., \& Eagles C. (1996). Acute effects of beta blockade and exercise on mood and anxiety. British of Journal of Sports Medicine, 30, 238-242.

Heath, B. H., \& Carter, J. E. L. (1967). A modified somatotype method. American Journal of Physical Anthropology, 27, 57-74.

Hedelin, R., Wiklund U., Bjerle P., \& Henriksson-Larsen, K. (2000). Pre and post season heart rate variability in adolescent cross-country skiers. Scandinavian Journal of Medicine and Science in Sports, 10, 298-303

Heijsman, S. M., Koers, N. F., Bocca, G., van der Veen, B. S., Appelhof, M., \& Kamps AW. (2012). Non-invasive measurement of adrenal response after standardized exercise tests in prepubertal children. J Pediatr Endocrinol Metab., 25(5-6):471-8.

lellamo, F., Pigozzi, F., Spataro, A., Lucini, D., \& Pagani, M. (2004). Twave and heart rate variability changes to assess training in worldclass athletes. Medicine and Science in Sports and Exercise, 36, 1342-1346.

Jacks DE, Sowash J, Anning J, McGloughlin T, Andres F. Effect of exercise at three exercise intensities on salivary cortisol. J Strength Cond Res 2002: 16: 286-289.

Jammes, Y., Arbogast, S., Faucher, M., Montmayeur, A., Tagliarini, F., \& Robinet, C. (2001). Interindividual variability of surface EMG changes during cycling exercise in healthy humans. Clinical Physiology, 21, 556-560.

Kalat, J. W. (2004). Biological Psychology (8th ed.). Canadá: Wadsworth, a division of Thomson Learning.

Kiecolt-Glaser, J., McGuire, L., Robles, Th., \& Glaser, R. (2002). Emotions, morbidity, and mortality: New perspectives from psychoneuroimmunology. Annual Review of Psychology, 53, 83-107.

Koelstra, S., Muhl, C., Soleymani, M., Lee, J.-S., Yazdani, A., Ebrahimi, T., Patras, I. (2011). DEAP: A database for emotion analysis using physiological signals [In press]. IEEE Transactions on Affective Computing. Special Issue on Naturalistic Affect Resources for System Building and Evaluation, 1-15. Recuperado de http://www.eecs.qmul. ac.uk/mmv/datasets/deap/doc/tac_special_issue_2011.pdf

Lago-Peñas, C., Casais, L., Dellal, A., Rey, E., \& Domínguez, E. (2011). Anthropometric and physiological characteristics of young soccer players according to their playing positions: relevance for competition success. J Strength Cond Res, 25(12), 3358-67. doi: 10.1519/JSC.0b013e318216305d.

Leicht, A.S., Allen, G.D., \& Hoey, A.J. (2003). Influence of intensive cycling training on heart rate variability during rest and exercise. Canadian Journal of Applied Physiology, 28, 898-909.

López-Torres, M., Torregrosa, M., \& Roca, R. (2007). Características del "Flow", ansiedad y estado emocional, en relación con el rendimiento de deportistas de élite. Cuadernos de Psicología del Deporte, 7(1): 25-44.

Lusa Cadore, E., Lhullier, F. L., Arias Brentano, M., Marczwski Da Silva, E., Bueno Ambrosini, M., Spinelli, R., Ferrari Silva, R., \& Martins Kruel, L. F. (2009). Salivary hormonal responses to resistance exercise in trained and untrained middle-aged men. I Sport Med Phys Fit, 49, 301-307.

Lyndsey, D. B. (1951). Emotion. En: S.S. Stevens (Ed.). Handbook of Experimental Psychology (pp. 473-516). New York: Wiley. 
El dilema de lo orgánico y lo social en las emociones deportivas

José Ingnacio Alonso Roque, Juan luis Yuste lucas, Gemma María Gea García y Nuria

UReña Ortín

MacLean, P. D. (1975). Sensory and perceptive factors in emotional function of the triune brain. En: R. G. Grenell \& S. Gabay (Eds.) Biological Foundations of Psychiatry. Vol. 1 (pp. 177-198). New York: Raven Press.

Marfell-Jones, M., Olds, T., Stewart, A., \& Carter, L. (2006). International standards for anthropometric assessment. Potchefstroom: ISAK.

Márquez, R., \& Garatachea, N. (2013). Relación entre actividad física y salud mental: El papel del ejercicio entre personas con depresión. En Márquez, R., y Garatachea, N. (Ed.), Actividad física y salud (pp. 99-110). Madrid: Díaz de Santos.

Martinsen, E.W. (2004). Physical activity and depression: clinical experience. Acta Psychiatrica Scandinavica, 89, 23-27.

Matthew-Lee, C., Wood, R., \& Welsch, M. (2003). Influence of short term endurance exercise training on heart rate variability. Medicine \& science in sports \& exercise, 35(6), 961-9.

Minetto, M. A., Lanfranco, F., Baldi, M., Termine, A., Kuipers, H., Ghigo, E., \& Rainoldi, A. (2007). Corticotroph axis sensitivity after exercise: comparison between elite athletes and sedentary subjects. J Endocrinol Invest, 30, 215-223.

Moreira, A., Arsati, F., de Oliveira Lima Arsati, Y. B., da Silva, D. A., \& de Arau' jo, V. C. (2009). Salivary cortisol in top-level professional soccer players. Eur J App/ Physiol, 106, 25-30.

Mourot, L., Bouchaddi, M., Perrey, S., Rouillon, J.D., \& Regnard, J. (2004). Quantitative poincare plot analysis of heart rate variability: effect of endurance training. European Journal of Applied Physiology, 91, 19-87.

Nuissier, F., Chapelot, D., Vallet, C., \& Pichon, A. (2007). Relations between psychometric profiles and cardiovascular autonomic regulation in physical education students. Eur J Appl Physiol, 99(6), 615-22. Doi: 10.1007/s00421-006-0385-4.

Oatley, K. (1992). Bestlaid schemes: The psychology of emotions. New York: Cambridge University Press.

Olds, J., \& Milner, P. (1954). Positive reinforcement produced by electrical stimulation of septal area and others regions of rat brain. Journal of Comparative and Physiological Psychology, 47, 419-427.

Paffenbarger, R. S., Lee, I. M., \& Leung, R. (2004). Physical activity and personal characteristics associated with depression and suicide in American college men. Acta Psychiatrica Scandinavica, 89, 16-22.

Pailhez, G., Rodríguez, A., Ariza, J., Palomo, A. L., y Bulbena, A. (2009). Somatotipo y esquizofrenia. Un estudio caso-control. Actas Esp Psiquiatr, 37(5), 258-266.

Palmero, F. (1996). Aproximación biológica al estudio de la emoción. Anales de Psicología, 12, 61-86.

Papez, J. W. (1937). A proposed mechanism of emotion. Journal of Neuropsychiatry and Clinical Neurosciences, 7, 103-112.

Picard, R. W. (2000). Affective Computing. Cambridge, MA: MIT Press.

Pichot, V., Roche, F., Gaspoz, J.M., Enolras, F., Antoniadis, A., Minimi, P., Costes, F., Busso, T., Lacour, J.R., \& Barthelemy, J.C. (2000). Relation between heart rate variability and training load in middle distance runners. Medicine and Science in Sport and Exercise, 32, 1729-1736. 
Pigozzi, F., Alabiso, A., Parisi, A., Disalvo, B., Di Luigi, L., Sparato, A., \& lellamo, F. (2001). Effects of aerobic exercise training on $24 \mathrm{hr}$ profile of heart rate variability in female athletes. Journal of Sports Medicine and Physical Fitness, 41, 101-107.

Pinna, S., Van Den Bergh, B. R. H., Van Calster, B., \& Van Huffel, S. (2008). Relación del perfil del cortisol salival en adolescentes de 14-15 años de edad, con la depresión, ansiedad y reactividad emocional. Revista Mexicana de Psicología, 25(2), 249-258.

Ramos, V., Piqueras, J. A., Martínez, A. E., \& Oblitas, L. A. (2009). Emoción y cognición: Implicaciones para el tratamiento. Terapia Psicológica, 27(2), 227-237.

Raschka, C., \& Wolthausen, C. (2007). Comparison of somatotype differences of soccer and handball players based on the methods of German and Anglo-American schools of constitutional biology. Anthropol Anz, 65(3), 303-16.

Raschka, C., \& Zanellato, S. (2003). Sports anthropology investigation on female participants of the German Sports-Aerobic-Masters compared to non-sports participating young females. Anthropol Anz, 61(4), 461-72.

Rodas, G., Pedret, C., Ramos, J., \& Capdevila, L. (2008). Variabilidad de la frecuencia cardiaca: conceptos, medidas y relación con aspectos clínicos (I). Archivos de Medicina del Deporte, 123, 41-47

Salovey, P., Rothman, A., Detweiler, J., \& Steward, W. (2000). Emotional states and physical health. American Psychologist, 55, 110-121.

Schelling, X., Calleja, J., \& Terrado, N. (2013). Variación de la testosterona y el cortisol en relación al estado de ánimo en jugadores de baloncesto de élite. International Journal of Sport Science, IX(34), 342-359.

Sheldon, W. H. (1960). Las variedades del temperamento: Psicología de las diferencias constitucionales. Buenos Aires: Paidos

Silverthorn, D. U. (2009). Fisiología humana. Un enfoque integrado. Buenos Aires: Panamericana.

Task Force of the European Society of Cardiology and The North American Society of Pacing and Electrophysiology. (1996). Heart Rate Variability - standards of measurement, physiological interpretation, and clinical use. European Heart Journal, 17, 354-381.

Vidales, I. (1978). Psicología General (11 a ed.). México, D. F.: Limusa.

Wilber, R. L., \& Pitsiladis, Y. P. (2012). Kenyan and Ethiopian distance runners: what makes them so good? Int J Sports Physiol Perform, 7(2), 92-102.

Wise, R. A. (1982). Neuroleptics and operant behaviour: The anhedonia hypothesis. Behavioral and Brain Sciences, 5, 39-87.

Wittaker, J. O. (1987). Psicología (4a ed.). México, D. F.: Interamericana.

\section{Agradecimientos}

Estos estudios han recibido financiación del Ministerio de Economía y Competitividad, Secretaría de Estado de Investigación, Desarrollo e Innovación de España a través del proyecto I+D+i Juegos Deportivos Psicomotores y de Cooperación DEP2010-21626-C03-01. 
